\title{
Microglia Enhance Synapse Activity to Promote Local Network Synchronization
}

Ryohei Akiyoshi, ${ }^{1,2}$ - $H i r o a k i$ Wake, ${ }^{3,6,7}$ Daisuke Kato, ${ }^{1,3}$ Hiroshi Horiuchi, ${ }^{1}$ Riho Ono, ${ }^{3}$ Ako Ikegami, ${ }^{3}$ Koichiro Haruwaka, ${ }^{1,2,3}$ Toshiaki Omori, ${ }^{4}$ (1) Yoshihisa Tachibana, ${ }^{3}{ }^{\circledR}$ Andrew J. Moorhouse, ${ }^{5}$ and (D) Junichi Nabekura ${ }^{1,2,6}$

https://doi.org/10.1523/ENEURO.0088-18.2018

${ }^{1}$ Division of Homeostatic Development, National Institute for Physiological Sciences, National Institutes of Natural Sciences, Okazaki, Japan 444-8585, ${ }^{2}$ Department of Physiological Sciences, the Graduate School for Advanced Study, Hayama, Japan 240-0193, ${ }^{3}$ Division of System Neuroscience, Kobe University Graduate School of Medicine, Kobe, Japan 650-0017,

${ }^{4}$ Department of Electrical and Electronic Engineering, Graduate School of Engineering, Kobe University, Kobe, Japan 657-8501, ${ }^{5}$ School of Medical Sciences, University of New South Wales, Sydney, Australia 2052, ${ }^{6}$ Core Research for Evolutional Science and Technology, Japan Science and Technology Agency, Saitama, Japan 100-0004, and ${ }^{7}$ Precursory Research for Embryonic Science and Technology, Japan Science and Technology Agency, Saitama, Japan 100-0004

\section{Visual Abstract}

\section{Microglia contact \\ physiologically synapse activity $\downarrow$ pathologically synapse activity $\rightarrow$}
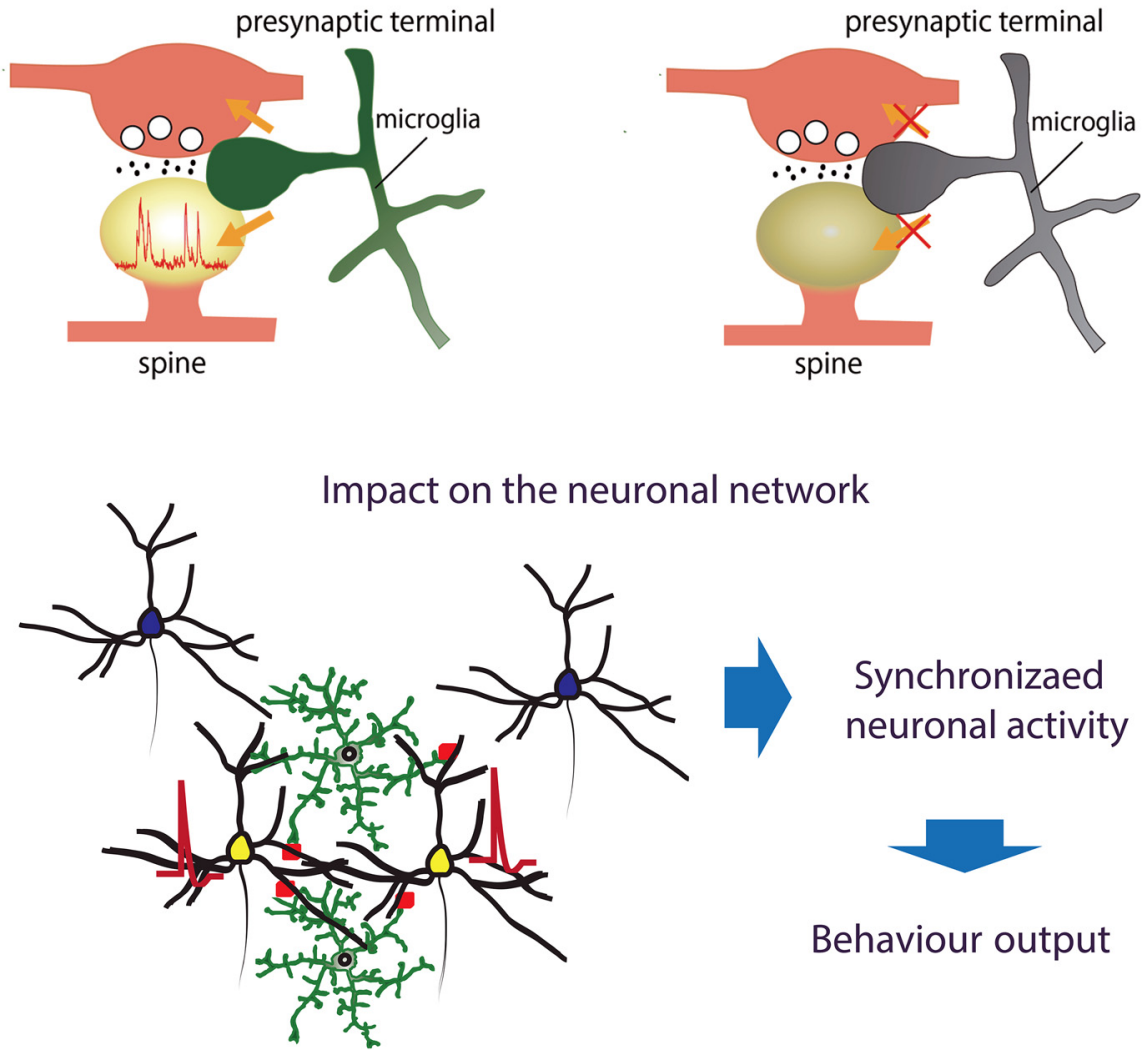

Synchronizaed neuronal activity

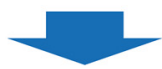

Behaviour output 


\section{Significance Statement}

Microglia, the sole immune cells in the central nervous system, make frequent contacts with synapses on dendritic spines, but the functional significance of these contact has remained elusive. In this study, we use in vivo two photon imaging and demonstrate that microglia contact on spines increases synaptic activity. This microglia-induced increase in synaptic activity enhances the synchronization of neuronal populations. This increase synchrony is inhibited by microglial activation, proving a possible mechanistic basis for how immune status may impact on neural circuit function.

Microglia are highly motile immunoreactive cells that play integral roles in the response to brain infection and damage, and in the progression of various neurological diseases. During development, microglia also help sculpt neural circuits, via both promoting synapse formation and by targeting specific synapses for elimination and phagocytosis. Microglia are also active surveyors of neural circuits in the mature, healthy brain, although the functional consequences of such microglia-neuron contacts under these conditions is unclear. Using in vivo imaging of neurons and microglia in awake mice, we report here the functional consequences of microgliasynapse contacts. Direct contact between a microglial process and a single synapse results in a specific increase in the activity of that contacted synapse, and a corresponding increase in back-propagating action potentials along the parent dendrite. This increase in activity is not seen for microglia-synapse contacts when microglia are activated by chronic lipopolysaccharide (LPS) treatment. To probe how this microglia-synapse contact affects neural circuits, we imaged across larger populations of motor cortical neurons. When microglia were again activated by LPS (or partially ablated), there was a decrease in the extent to which neuronal activity was synchronized. Together, our results demonstrate that interactions between physiological or resting microglia and synapses in the mature, healthy brain leads to an increase in neuronal activity and thereby helps to synchronize local populations of neurons. Our novel findings provide a plausible physical basis for understanding how alterations in immune status may impact on neural circuit plasticity and on cognitive behaviors such as learning.

Key words: calcium imaging; in vivo; microglia; synapse; two photon

\section{Introduction}

Microglia are highly motile immune effector cells in the brain that respond to neuronal infection and damage by converting from a resting or physiologic phenotype, to an activated or reactive phenotype. This reactive phenotype is associated with morphologic changes, migration and proliferation, release of inflammatory and neuroactive molecules, and ultimately phagocytosis of damaged neuronal elements (Kettenmann et al., 2011). Such microglia activation is a hallmark of the pathogenesis of neurodegenerative diseases such as Alzheimer's disease, Parkinson's disease, and amyotrophic lateral sclerosis (Cunningham,

Received March 6, 2018; accepted September 22, 2018; First published October 11, 2018.

The authors declare no competing financial interests.

Author contributions: H.W., J.N., A.J.M., and R.A. designed research; D.K., H.W., H.H., R.O., A.I., and R.A performed research; D.K., K.H., H.H., T.O., A.I., R.O., Y.T., and H.W., analyzed data; H.W., J.N., and A.J.M. wrote the paper. Correspondence should be addressed to either of the following: Dr. Hiroaki Wake, Professor Division of System Neuroscience, Kobe University Graduate School of Medicine, 7-5-2 Kusunoki-cho, Chuo-ku, Kobe 650-0017, Japan, E-mail: hirowake@med.kobe-u.ac.jp; or Dr. Junichi Nabekura, Professor Division of Homeostatic Development, National Institute for Physiological Sciences, National Institutes of Natural Sciences, 38 Myodaiji, Okazaki, 444-8585, Japan, E-mail: nabekura@nips.ac.jp.

https://doi.org/10.1523/ENEURO.0088-18.2018

Copyright $(\odot 2018$ Akiyoshi et al.

This is an open-access article distributed under the terms of the Creative Commons Attribution 4.0 International license, which permits unrestricted use, distribution and reproduction in any medium provided that the original work is properly attributed.
2013). Whether microglia activation occurs early in the disease pathogenesis to trigger some aspects of neuronal dysfunction is less clear. A broader question is to what extent disruptions in the interactions between physiologic microglia and neural circuits, such as may occur in response to microglial activation, impacts on neuronal homeostasis and cognitive performance (Salter and Beggs, 2014; Estes and McAllister, 2015; Kipnis, 2016; Tay et al., 2017). These physiologic microglia are far from resting, they actively survey the brain parenchyma with their processes making frequent and direct contacts with neuronal synapses (Nimmerjahn et al., 2005; Wake et al., 2009). This contact between microglial processes and the various neuronal elements appears to occur in an activity dependent fashion (Dissing-Olesen et al., 2014; Eyo et al., 2014), but the consequences of this interaction for neural circuit homeostasis and plasticity in the mature, healthy brain are not fully understood. Microglia neuronal contacts can physically sculpt neural circuits, through phagocytosing weaker or inactive synapses during development and after injury (Schafer et al., 2012), and through promoting neuronal synapse and/or spine formation either directly or indirectly (Parkhurst et al., 2013; Miyamoto et al., 2016). However, the acute effects of microglianeuron contacts on neural activity are less clear. In immature zebrafish neurons, microglia-neuron contacts can reduce neuronal activity (Li et al., 2012), and we proposed that interactions between physiologic microglia and neuronal synapses modulates neural circuit activity in the 
mature, healthy mammalian brain. To examine this hypothesis, we combined in vivo imaging of physiologic and activated microglia with imaging of neuronal activity in awake mice, at both the single synapse level and across neural circuits. Our results demonstrate that physiologic microglia can selectively enhance the activity of synapses and neurons that they contact. We show that this microglia-neuron contact results in an increase in the synchronization of activity across local neuronal populations. Our results have marked implications for the understanding of how immune status can impact on neural network activity and cognitive function, and suggest that microglia could potentially play a primary role in cognitive dysfunction associated with aging and psychiatric diseases.

\section{Materials and Methods}

\section{Animals and microglia ablation or activation}

All animal experiments were approved by the Animal Research Committees. Mice were given free access to food and water in a 12/12 h light/dark cycle, and we used male mice for all experiments. To image microglia, we used ionized $\mathrm{Ca}^{2+}$-binding adapter molecule 1 (Iba1)enhanced green fluorescent protein (EGFP) transgenic mice, which expresses EGFP under the control of the lba1 promoter, which is specific for microglia and macrophages (Hirasawa et al., 2005). For microglia ablation experiments, we crossed lba1-tetracycline transactivator (Iba1-tTA) mice (Tanaka et al., 2012) with tetracycline operator-diphtheria toxin A (tetO-DTA) mice (Stanger et al., 2007). Withdrawal of doxycycline (Dox-off) in the feed of these mice leads to selective expression of the DTA in microglia. All transgenic mice were derived from the C57BL/6J strain. Mice were reared with standard chow containing Dox $0.1 \mathrm{~g} \mathrm{~kg}^{-1}$. This was replaced with Dox-free standard chow from seven weeks after birth to induce transgene induction, a time corresponding to $7 \mathrm{~d}$ before the first imaging experiment. Lipopolysaccharide (LPS; Funakoshi) was used to pharmacologically activate microglia, and was injected daily (1.0 mg kg ${ }^{-1}$, i.p.) for 9 d. Control mice received intraperitoneal saline injections with the same dosing schedule. Imaging was performed on day 4 and day 9 during the LPS or saline injection. In an additional cohort used to examine time course and recovery, chronic imaging was performed before LPS, and 1 and $4 \mathrm{~d}$ after daily LPS injections, and again at $7 \mathrm{~d}$ after stopping LPS.

\section{Surgery and adeno-associated virus (AAV) injection}

All mice were anesthetized by ketamine $(74 \mathrm{mg} / \mathrm{kg}$, i.p.) and xylazine $(10 \mathrm{mg} / \mathrm{kg}$, i.p.). The skin was disinfected with $70 \%$ (w/vol) ethanol, the skull was exposed and cleaned, and a custom-made head plate was firmly attached using dental cement (Fujiryu-to BC; GC, Bistite II; Tokuyama Dental). The surface of the intact skull was subsequently coated with an acrylic-based dental adhesive resin cement (Super bond; Sun Medical) to avoid drying. Mice were allowed to recover for $1 \mathrm{~d}$ before open skull surgery and/or viral injection.
Under isoflurane (1\%) anesthesia, a circular craniotomy ( $\sim 2 \mathrm{~mm}$ in diameter) was performed (Masamizu et al., 2014) over the left primary motor cortex (centered at 0.2 $\mathrm{mm}$ anterior and $1 \mathrm{~mm}$ lateral from bregma). To visualize neuronal activity in L2/3 pyramidal neurons, a rAAV2/1Syn-GCaMP6 AAV (UPenn Vector Core; $9.0 \times 10^{12}$ vector genomes $/ \mathrm{ml}$, diluted $1: 5$ in PBS) was injected at three sites in L2/3 of the cortex using a glass pipette (tip diameter: $10 \mu \mathrm{m}$ ), which was left in the brain for an additional 10 min to avoid backflow. For structural and $\mathrm{Ca}^{2+}$ imaging of apical dendrites and spines of layer 5 (L5) pyramidal neurons, a mixed AAV [UPenn Vector Core; AAV2/1-SynFLEX-GCaMP6: $1.09 \times 10^{13}$ vector genomes/ml, AAV2/ 1-CAG-FLEX-Tdtomato: $7.6 \times 10^{12}$ vector genomes $/ \mathrm{ml}$, and AAV2/1-CaMKII -Cre: $2.94 \times 10^{13}$ vector genomes $/ \mathrm{ml}$ (1: 10 000) diluted in PBS, three sites] solution was similarly injected into L5 of the cortex. After the injection, 2\% (w/v) agarose L (Nippon Gene) was placed over the brain surface and a glass window comprised of two coverslips (Matsunami Glass) sealed with an ultraviolet curable adhesive (NOR-61, Norland) was implanted over the craniotomy. The edges of the cranial window were sealed with dental cement and dental adhesive resin cement.

\section{Two-photon imaging}

$\mathrm{Ca}^{2+}$ imaging of $\mathrm{L} 2 / 3$ pyramidal neurons was conducted using a LSM 7 MP system (Zeiss) and a modelocked Ti:sapphire Chameleon Ultra II laser (Coherent) tuned to $950 \mathrm{~nm}$, with a $20 \times$ objective (XLPlan, NA of 1.0; Zeiss). Fluorescence was separated by a $570-\mathrm{nm}$ dichroic mirror with 495-550 nm (green channel: for EGFP fluorescence detection) and 570-630 nm (red channel: for tdTomato fluorescence detection) emission filters and was collected using GaAsP photomultiplier tubes (Hamamatsu Photonics). The laser intensity was $5-30 \mathrm{~mW}$. The imaged fields were $424.27 \times 424.27 \mu \mathrm{m}$, at a depth of $150-200$ $\mu \mathrm{m}$ below the cortical surface. The pixel size was 0.830 $\mu \mathrm{m}$. Frame duration was $390 \mathrm{~ms}$, and continuous 1000frame imaging was repeated for each field for 10-30 min. For imaging the apical dendrites (and their spines) of L5 pyramidal neurons in awake mice, the image plane was focused at a depth within $50 \mu \mathrm{m}$ from the cortical surface (L1). The image fields were $28.28 \times 28.28 \mu \mathrm{m}$, with a pixel size of $0.055 \mu \mathrm{m}$ and a frame duration of $242 \mathrm{~ms}$. Continuous 3000-frame imaging was repeated in each field for $12 \mathrm{~min}$.

\section{Image analysis}

Analyses were performed using an ImageJ plug-in $(1.37 \mathrm{v} ; \mathrm{NIH}$ ) and programs written in MATLAB (version 8; MathWorks). Movies were corrected for focal plane displacements using TurboReg (Thevenaz et al., 1998). To quantify neural activity, regions of interest (ROIs) around a cell were determined using a semi-automated algorithm that correlated the fluorescence intensity between adjacent pixels to define the cell edge, and ROls were confirmed by visual inspection. Note this procedure only detects neurons with fluorescent responses. For time lapse imaging of interactions between microglia and neurons, ROls were manually defined around single spines or around dendrites. The fluorescence within a ROI was 
quantified for each image and averaged over time, with background fluorescence subsequently subtracted from this averaged fluorescence. Slow timescale changes in fluorescence were removed from the fluorescence time series values using the method of (Dombeck et al., 2007), in which the distribution of fluorescence in a \pm 15 -s interval around each sample time point was determined, and the 8th percentile was subtracted. For identification of significant transients in each ROI, the baseline and SD $(\sigma)$ were calculated from manually selected large transientsfree regions. A fluorescent $\mathrm{Ca}^{2+}$ transient was detected if the fluorescent intensity $(\mathrm{Fl})$ exceeded $4 \mathrm{SDs}(\sigma)$ above the mean baseline. The correlation coefficient (C.C.) was obtained by quantifying the time in which the $\mathrm{Ca}^{2+}$ transient was at its peak value, and grouping these times into 242-ms bins.

ImageJ was used to quantify contacts between GFPlabeled microglia and tdTomato-labeled spines. Images were manually screened across all time points and across different Z planes to detect contacts as overlap between the red (spine) and the green (microglia) fluorescent channels, after determining the baseline thresholds for each channel respectively. Real contacts were defined only if the red and the green fluorescence overlap occurred in at least two $Z$ sections.

\section{Immunohistochemistry}

To quantify microglia morphology, mice were transcardially perfused with $4 \%$ paraformaldehyde in phosphate buffer (PB; pH 7.4), and brains were excised and fixed overnight in the same solution. Fixed brains were equilibrated in a $30 \%$ sucrose solution in PB, and cut with a microtome (Leica Microsystems) into $30-\mu \mathrm{m}$-thick sections. Sections containing primary motor cortex were incubated overnight with primary antibody (anti-lba1 antibody, 1:500 diluted in 10\% normal goat serum/PBST; Wako) at $4^{\circ} \mathrm{C}$, followed by 1 -h incubation with secondary antibody (Alexa Fluor 488-conjugated donkey anti-mouse; Invitrogen). Fluorescent signals were detected with a LSM 5 DUO system (Zeiss).

\section{Image analysis for microglia morphology}

Iba1-labeled fixed brain slices were imaged on a confocal microscope with an Argon laser operating at a wavelength of $488 \mathrm{~nm}$. A laser scanning system (Carl Zeiss Microlmaging, Inc.) with water-immersion objective (20×, 1.0 NA, Zeiss) was used for image acquisition. $Z$ stack images $(512 \times 512$ pixels, $0.198-\mu \mathrm{m}$ pixel-1, $0.5-\mu \mathrm{m}$ Z-step) were used for morphologic analysis of microglia. The extent of ramification of microglia processes was quantified by circumscribing the area of the distal portions of microglial processes using the segmented line tool in ImageJ. Microglia size was measured by circumscribing the cell body using an intensity threshold tool in ImageJ. Morphometric quantification was done with Neurolucida, performing a Sholl analysis of process number and length with respect to standard distances from the soma.

\section{Data and statistics}

Data are presented as mean \pm SEM. Unpaired, paired, and one-sample $t$ test, Wilcoxon rank sum tests, and
Pearson's correlation tests were used to test for statistical significance as indicated. When analyzing the $\mathrm{Ca}^{2+}$ imaging data in both soma and spines, we first performed the D'Agostino and Pearson normality test, which indicated data were not normally distributed.

\section{Results}

\section{Physiologic microglial contact with synapses enhances neuronal activity}

In the healthy, mature cortex, quiescent or physiologic microglial processes are highly motile and survey the neuronal environment (Nimmerjahn et al., 2005; Wake et al., 2009). These motile processes specifically and directly contact synapses, and the frequency and extent of these contacts depends on neuronal activity (Wake et al., 2009; Tremblay et al., 2010; Li et al., 2012). To probe the functional consequences of these microgliasynapse contacts, we simultaneously visualized neuronal activity and microglial processes, using time-lapse in vivo imaging in awake mice. Microglia were identified by EGFP fluorescence driven by a microglia-specific promoter (using Iba1-EGFP mice), while neuronal activity was monitored using injection of an AAV expressing the $\mathrm{Ca}^{2+}$ sensor, GCaMP6f, along with the reporter construct tdTomato (Fig. 1A). As we reported previously (Wake et al., 2009), microglial processes made direct and brief $(\approx 5$ min) contacts with synapses located on dendritic spines (Fig. 1A). Using high resolution imaging we could detect and quantify localized $\mathrm{Ca}^{2+}$ transients within single dendritic spines, during which time (up to $12 \mathrm{~min}$ ) we sometimes observed a period of microglial contact. During the microglial contact period, the frequency of spine $\mathrm{Ca}^{2+}$ transients was significantly increased as compared to that either before or after contact [Fig. $1 A-C ; 0.020 \pm 0.005 \mathrm{~Hz}$ (during) vs $0.010 \pm 0.002 \mathrm{~Hz}$ (before), $p=0.033, n=20$ fields from 17 mice (Fig. 1B); $0.016 \pm 0.007 \mathrm{~Hz}$ (during) vs $0.004 \pm 0.003 \mathrm{~Hz}$ (after), $p=0.030, n=10$ fields from 10 mice (Fig. 1C), paired $t$ test]. The increase in spine activity was consistently seen, although variable in extent, and was clearly reversible on withdrawal of the microglial process from the spine (Fig. $1 A-C$ ).

$\mathrm{Ca}^{2+}$ transients occurring along the entire dendrite, and that were co-incident with dendritic spine transients, were also observed in these experiments, presumably reflecting back-propagating action potentials (BPs) originating from the neuronal soma. The frequency of these BPassociated dendritic $\mathrm{Ca}^{2+}$ transients was also increased during microglia contact onto synapses [Fig. 1D,E; 0.089 $\pm 0.011 \mathrm{~Hz}$ (during) vs $0.074 \pm 0.012 \mathrm{~Hz}$ (before), $p=$ $0.044, n=20$ fields from 17 mice (Fig. 1D); $0.093 \pm 0.017$ $\mathrm{Hz}$ (during) vs $0.063 \pm 0.017 \mathrm{~Hz}$ (after), $p=0.003, n=10$ fields from 10 mice (Fig. 1E), paired $t$ test], suggesting the increased spine activity associated with microglia contact also resulted in an increase in excitability of the neuron at the soma (Fig. 1D,E).

To examine how microglial processes may select particular spines to be contacted, we compared (in different experiments from above) the basal rate of $\mathrm{Ca}^{2+}$ transients in spines and dendrites in contacted and non-contacted spines within the same image fields (Fig. 1F). Those 
A
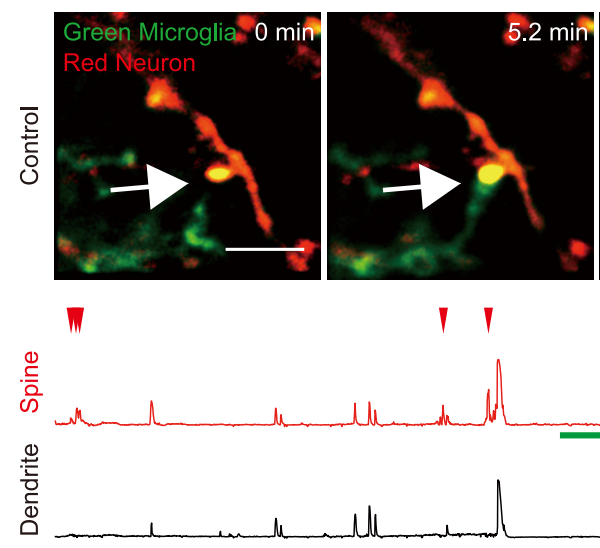

D

E

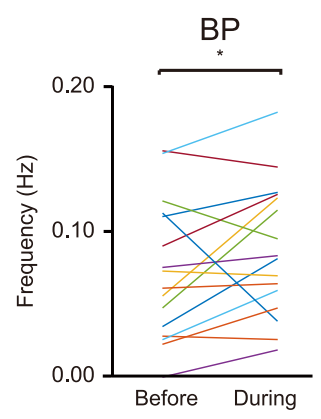

B

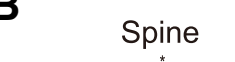

C

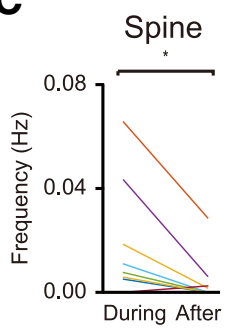

II
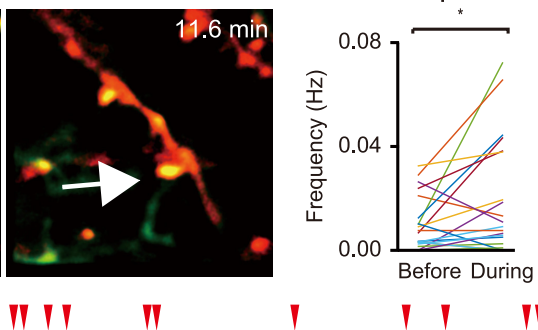

II

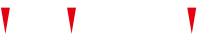
Nhurer and

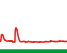

glia Contact

Microglia Contac

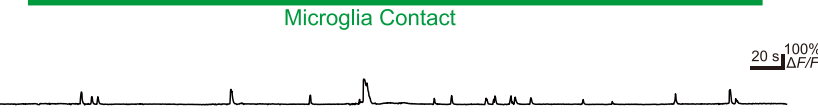

F

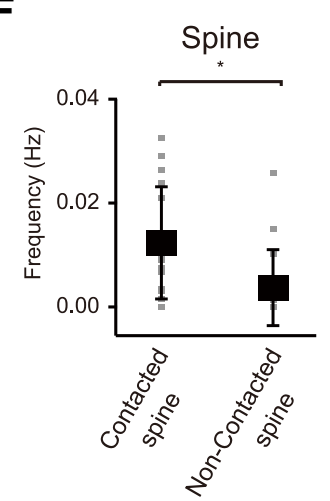

G

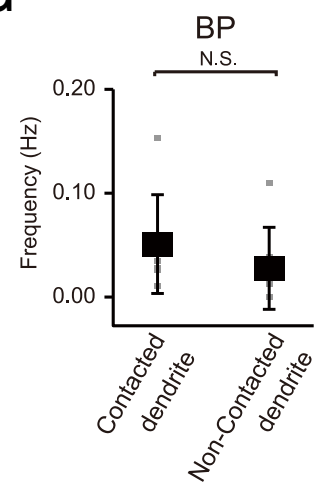

Figure 1. Enhanced synapse activity follows microglia contact. $\boldsymbol{A}$, top, Fluorescent image of a dendrite in in $L 2 / 3$ of the primary motor cortex showing the simultaneous imaging of spines and their parent dendrite (tdTomato; red) and microglia (EGFP, green). Scale bar: $5 \mu \mathrm{m}$. The image panels show microglial process contacting a spine (center). Lower traces, Activity in the neuronal compartments was measured with GCaMP6f and quantified as $\Delta F / F$. Sample $\mathrm{Ca}^{2+}$ traces from a spine and its parent dendrite before, during, and after microglia contact are shown. Putative backpropagating action potentials cause synchronous responses in both dendrite and spine, whereas presumed local synaptic activity results in spine transients only. Note the enhanced local (spine) synaptic activity with microglial contact. The white arrow indicate the spine contacted by microglia process. $\boldsymbol{B}, \boldsymbol{C}$, The frequency of local spine $\mathrm{Ca}^{2+}$ transients were increased during microglia contact periods relative to those before $(\boldsymbol{B})$ or after $(\boldsymbol{C})$ contact $[\boldsymbol{B}: 0.020 \pm 0.005 \mathrm{~Hz}$ (during) vs $0.010 \pm 0.002$ $\mathrm{Hz}$ (before), $p=0.033, n=20$ fields from 17 mice; $\boldsymbol{C}$ : $0.016 \pm 0.007 \mathrm{~Hz}$ (during) vs $0.004 \pm 0.003 \mathrm{~Hz}$ (after), $p=0.030, n=10$ fields from 10 mice, paired $t$ test]. $\boldsymbol{D}, \boldsymbol{E}$, The frequency of back propagating action potentials reflected as dendritic $\mathrm{Ca}^{2+}$ transients was increased during the period of microglia-synapse contact, relative to that before $(\boldsymbol{D})$ or after $(\boldsymbol{E})$ contact $[\boldsymbol{D}: 0.089 \pm 0.011 \mathrm{~Hz}$ (during) vs $0.074 \pm 0.012$ $\mathrm{Hz}$ (before), $p=0.044, n=20$ fields from 17 mice; $\boldsymbol{E}$ : $0.093 \pm 0.017 \mathrm{~Hz}$ (during) vs $0.063 \pm 0.017 \mathrm{~Hz}$ (after), $p=0.003, n=10$ fields from 10 mice, paired $t$ test]. $\boldsymbol{F}$, Microglia were attracted to synapses with a higher basal (pre-contact) frequency of local $\mathrm{Ca}^{2+}$ responses (spines with microglia contact: $0.012 \pm 0.011 \mathrm{~Hz}$; spines without microglia contact: $0.004 \pm 0.007 \mathrm{~Hz}, p=0.004, n=16$ fields from 15 mice, Wilcoxon rank sum test). $\mathbf{G}$, In contrast, contact frequency did not depend on the basal frequency of back propagating action potentials (dendrites with microglia contact: $0.047 \pm 0.048 \mathrm{~Hz}$; dendrites without microglia contact: $0.028 \pm 0.040 \mathrm{~Hz}, p=0.32, n=7 \mathrm{fields}$ from seven mice, Wilcoxon rank sum test). Data are presented as the mean $\pm \mathrm{SEM} ; * p<0.05$.

spines that were contacted by microglia had significantly higher basal rates of $\mathrm{Ca}^{2+}$ transients before the microglia contacts (Fig. 1F; $0.012 \pm 0.011 \mathrm{~Hz}$ ) compared to spines without any apparent contacts (Fig. $1 F ; 0.004 \pm 0.007 \mathrm{~Hz}$, $p=0.004, n=16$ fields from 15 mice, Wilcoxon rank sum test). However, this higher basal rate was restricted to the spines, as the frequency of $\mathrm{Ca}^{2+}$ transients in the parent dendrite before microglia contact was not different from that in dendrites with spines that were not-contacted (Fig. $1 G$; dendrites with microglia contact; $0.047 \pm 0.048 \mathrm{~Hz}$, dendrites without microglia contact; $0.028 \pm 0.040 \mathrm{~Hz}, p$ $=0.32, n=7$ fields from seven mice, Wilcoxon rank sum test). Together, this suggests a very localized level of neuronal activity restricted just to the synapse influences microglial process contact. Such local activity may evoke an attractant signal with minimal spatial influence, as has been suggested previously (Wake et al., 2009; DissingOlesen et al., 2014).

After retracting from a dendritic spine, a microglial process continued surveying the local environment and could make a second contact with a different spine on the same dendrite (Fig. 2). This is consistent with microglia being attracted to more active synapses and with the localized region of surveillance of each microglia. However, the same spine could also be again contacted by a different microglial process (Fig. 2). As the frequency of contacts 
0
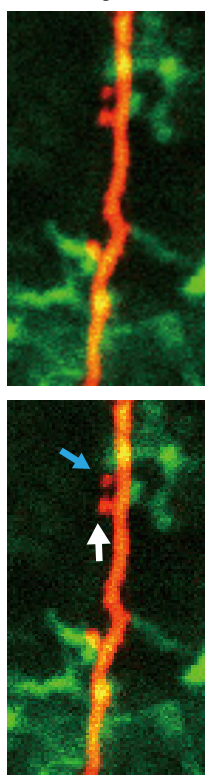

35
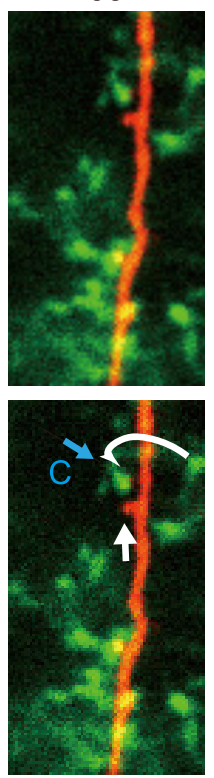

42
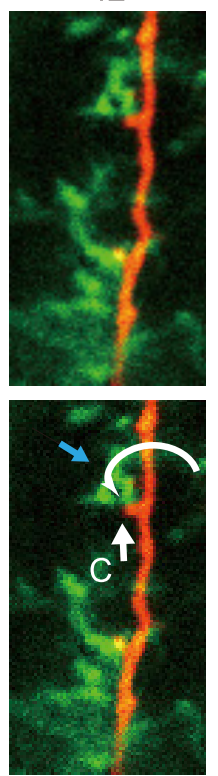

129
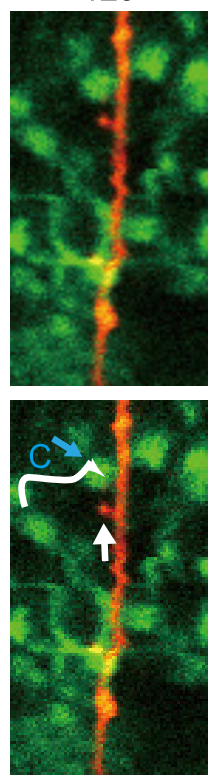

$194 \mathrm{~min}$
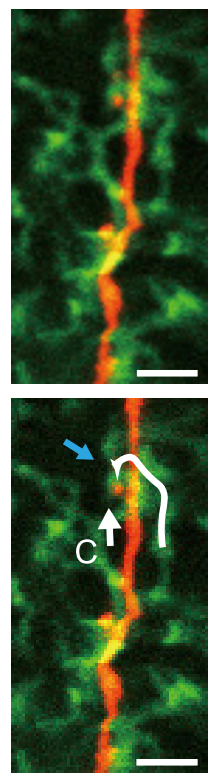

Figure 2. Specific physiologic microglial processes contact different spines while a single spine may be contacted by different microglia. Representative long-term simultaneous images of microglia (green) and of dendrites and spines (red), showing examples of microglial-synapse contacts during over three consecutive hours. The upper and lower panels show the same images with the lower panels annotated by white and blue straight arrows to indicate two different spines, and with a curved white arrow to indicate the trajectory of for a single microglial process as it contacts a spine (contact indicated by C). The upper spine (blue arrow) is contacted by one microglia at 35-42 min, and by a seemingly different microglia at $129 \mathrm{~min}$. At 42 and 194 min, the original microglia contacts the lower (white arrow) spine.

within the same image field was only about once per hour, subsequent longer-term imaging will be needed to quantify the patterns of contact by each single microglia.

\section{The physiologic microglial phenotype is necessary for synchrony of local network activity}

To examine how microglial contact-induced synapse modulation may affect neuronal circuit activity, we imaged activity in larger populations of neurons and compared results with mice with reduced microglia population. Ablation of microglia was achieved by crossing lba1-tTA mice (Tanaka et al., 2012) with TetO-DTA mice (Stanger et al., 2007) to establish an Iba1-tTA-TetO-DTA mouse colony. Dox-off from the chow of these mice causes selective expression of the DTA in microglia, resulting in a marked reduction in the microglia population. It has previously been shown that the levels of cytokines are not elevated with microglia ablation (Parkhurst et al., 2013). As expected, dox removal for $7 \mathrm{~d}$ resulted in a significant reduction in the density of microglia (Fig. $3 A, B$ ) from 121.3 $\pm 18.7 / \mathrm{mm}^{2}$ ( $n=7$ fields from three Dox on mice) to 87.4 $\pm 25.1 / \mathrm{mm}^{2}(n=10$ fields from three Dox-off mice; $p=$ 0.006 , unpaired $t$ test). To quantify neural circuit activity, we injected the GCaMP6f expressing AAV into these mice and quantified $\mathrm{Ca}^{2+}$ fluorescence across multiple neurons in L2/3 of the primary motor cortex (Fig. $3 C-F$ ). As shown in Figure $3 E$, the frequency of $\mathrm{Ca}^{2+}$ activity in L2/3 neurons from Dox on control mice was not significantly different from that in Dox off mice [Fig. 3E; Dox on $(n=269$ neurons from seven mice); $0.071 \pm 0.003 \mathrm{~Hz}$, Dox off $(n=$ 326 neurons from seven mice); $0.062 \pm 0.002 \mathrm{~Hz}, p=$
0.463, Wilcoxon rank sum test]. To quantify neuronal synchronization, we defined a C.C. between every individual pair of neurons within the image area in each mouse. The C.C. of activity in paired neurons in microglia ablated mice (Dox off for $7 \mathrm{~d}$ ) was reduced compared to control (Dox on) littermates [Fig. 3D; control mice $(n=$ seven mice); $0.28 \pm 0.006$, microglia ablated mice ( $n=7$ mice); $0.12 \pm 0.003, p=1.75 \times 10^{-171}$, Wilcoxon rank sum test]. Previous work in the M1 motor cortex has shown that correlations between activity of paired neurons varies as a function of the distance separating the neurons (Hira et al., 2013), with neurons closer together more likely to fire together. Hence, we plotted the C.C. against the distance between each of the neurons within the pair to examine if this relationship is changed in microglia ablated mice (Fig. 3F). In control mice, there was a weak inverse relationship between C.C. and separation distance $\left(r=-0.171, p=7.99 \times 10^{-30}\right.$, Pearson's correlation test), indicating that $L 2 / 3$ neurons located closer to each other are more likely to fire together. In contrast, this correlation was diminished in microglia ablated mice ( $r=-0.065, p=1.78 \times 10^{-8}$, Pearson's correlation test). To further validate this difference, we averaged the slope of the relationship between C.C. and neuronal separation distance in each mouse within the Dox on and off groups. The relationship between C.C. and neuronal separation was abolished by microglial ablation (Fig. 3F; Dox on: $n=$ $7, r=-0.175 \pm 0.045, p=0.0082$, Dox off: $n=7, r=$ $-0.048 \pm 0.036, p=0.227$, one-sample $t$ test). Together, our population imaging indicates that microglia ablation results in reduced synchronous firing of $L 2 / 3$ neurons, and 
A

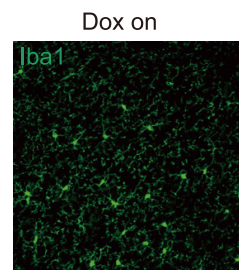

C

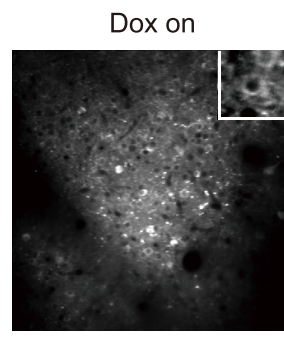

Dox off
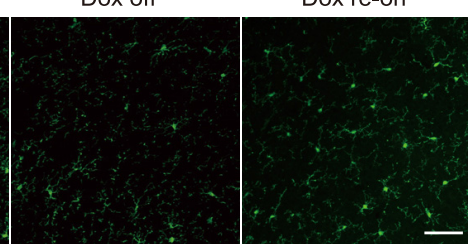

Dox re-on

B

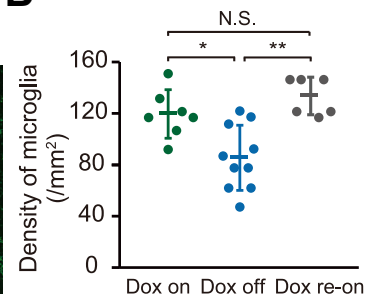

D
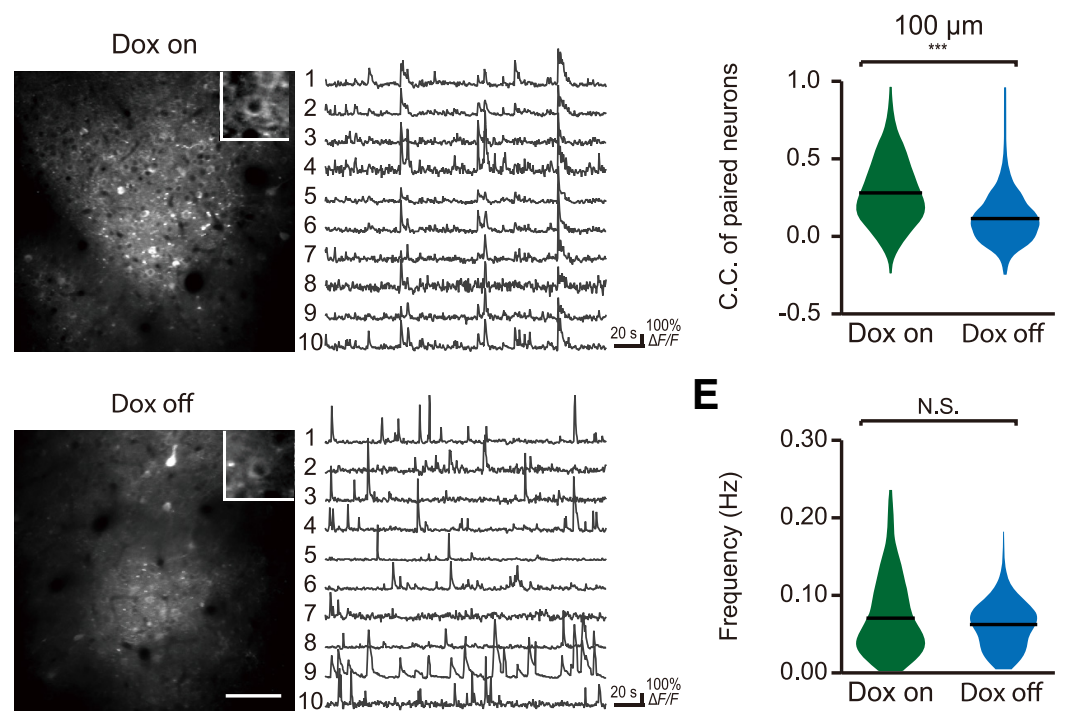

$\mathbf{F}$

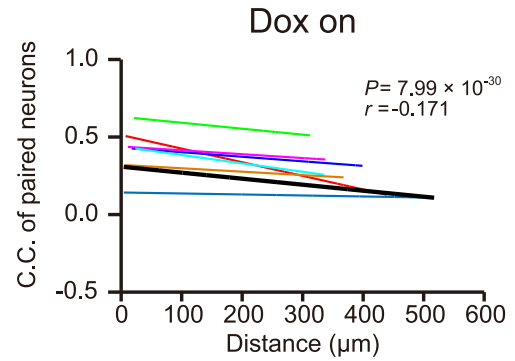

G

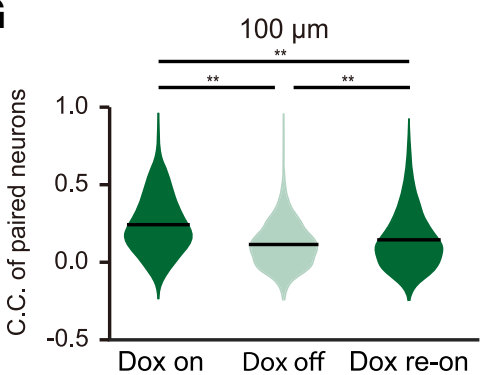

E

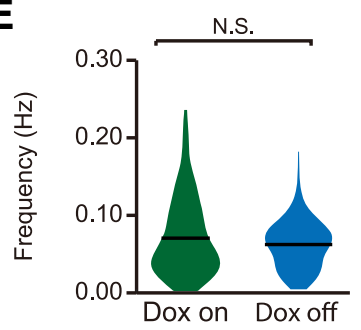

Dox off

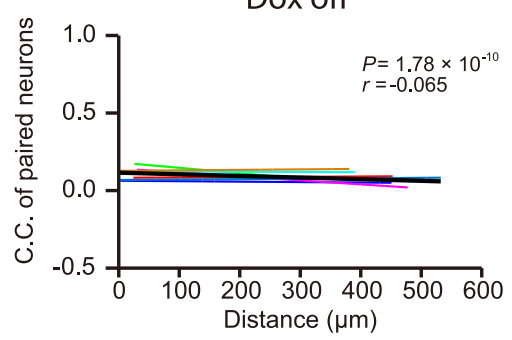

H

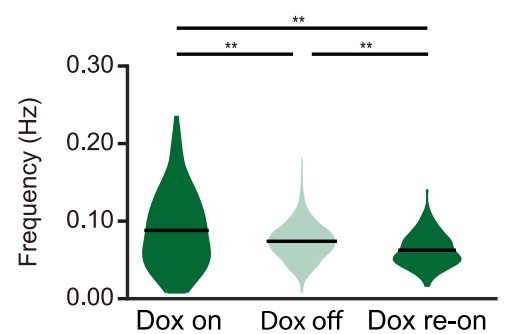

Figure 3. Decreased synchronization of evoked neural activity in microglia ablated mice. $\boldsymbol{A}$. Fluorescent (immunohistochemistry) images of Iba1-positive microglia in cortical sections of Dox on (left), Dox off (center), and re-Dox on mice showing reduced density of Iba1 (microglia)-positive cells in Iba1-tTA::tetO-DTA transgenic mice following $7 \mathrm{~d}$ of Dox withdrawal as compared with Iba1-tTA:: tetO-DTA transgenic mice with Dox maintained in the diet and in Dox-off mice after Dox had been returned to the diet for a week (Dox re-on). Scale bar in right panel: $50 \mu \mathrm{m}$. B, Genetic ablation of microglia was verified by quantification of microglia density. Dox-off mice showed a significant reduction in the density of microglia from $121.3 \pm 18.7 / \mathrm{mm}^{2}$ ( $n=7$ fields from three Dox on mice) to 87.4 $\pm 25.1 / \mathrm{mm}^{2}$ ( $n=10$ fields from three Dox-off mice; $p=0.006$, unpaired $t$ test). The density of microglia recovered to control levels following one week of Dox return to the diet (135.0 $\pm 14.5 / \mathrm{mm}^{2}, n=6$ fields from three Dox re-on mice; $p=0.0008$, unpaired $t$ test). C, left, Typical fields of view of L2/3 primary motor cortex neurons expressing GCaMP6f in Dox on mice (upper) and Dox off mice (lower). Right, Representative $\Delta F / F \mathrm{Ca}^{2+}$ traces from individual GCaMP6f-positive neurons from Dox on (upper) and Dox off mice 
continued

(lower). $\boldsymbol{D}$, Synchronous neuronal firing was estimated by measuring the proportion of neuron pairs in which $\mathrm{Ca}^{2+}$ transients were seen at the same time, and quantifying this as a C.C. The mean C.C. of paired neurons within $100 \mu \mathrm{m}$ of each other was lower in Dox off mice as compared with that in Dox on mice [Dox on mice $(n=$ seven mice); $0.28 \pm 0.006$, Dox off mice $(n=7$ mice); $0.12 \pm 0.003$, $p=1.75 \times 10^{-171}$, Wilcoxon rank sum test]. $\boldsymbol{E}$. There was no difference in the frequency of $\mathrm{Ca}^{2+}$ responses between Dox on and Dox off mice [Dox on ( $n=269$ neurons from seven mice); $0.071 \pm 0.003 \mathrm{~Hz}$, Dox off $(n=326$ neurons from seven mice); $0.062 \pm$ $0.002 \mathrm{~Hz}, p=0.463$, Wilcoxon rank sum test]. $\boldsymbol{F}$, The C.C.s in each pair of neurons was negatively correlated with the distance separating each pair of neurons in control (Dox on) mice (left). However, this negative correlation was absent in Dox off mice (right). Individual slope correlations from each mouse are shown by the thin colored regression lines, while the thicker black line shows the correlation obtained by fitting all the data simultaneously. The value of the slope of this group correlation and the $p$ value obtained from the Pearson's correlation test are shown in the top right of each graph, while averaged slope values obtained from each mouse are shown in this legend (control mice: $n=7, r=-0.171, p=7.99 \times 10^{-30}$; microglia ablated mice: $n=7, r=-0.065, p=1.78$ $\times 10^{-8}$, Pearson's correlation test; for seven Dox on mice, $r=-0.175 \pm 0.045, p=0.0082$, one-sample $t$ test; for seven Dox off mice, $r=-0.048 \pm 0.036, p=0.227$, one-sample $t$ test). $\mathbf{G}, \boldsymbol{H}$, In an additional cohort of mice, the C.C.s of neurons located within $100 \mu \mathrm{m}$ of each other $(\boldsymbol{G})$ and the neuron firing frequencies $(\boldsymbol{H})$ were measured during the doc on diet (left), following $7 \mathrm{~d}$ of Dox off diet (center) and again $42 \mathrm{~d}$ after returning to dox diet (dox re-on, right). Dox-off again reduced the C.C. which partially recovered after returning to $\operatorname{dox}\left[G: F_{(2,4926)}=200.21, p=9.56 \times 10^{-10}\right.$ (Dox on: $0.243 \pm 0.006$ vs Dox off: $0.115 \pm 0.003$ ), $p=3.15 \times 10^{-7}$ (Dox off: 0.115 \pm 0.003 vs Dox re-on: $0.145 \pm 0.005$ ), $p=9.56 \times 10^{-10}$ (Dox on: $0.243 \pm 0.006$ vs Dox re-on: $0.145 \pm 0.005$ ), one-way ANOVA followed by Tukey's test]. The neuronal firing frequencies in this cohort were decreased after Dox off and decreased further after returning to dox on $[\boldsymbol{H}$ : Dox on $(n=165$ neurons, four fields from two mice); $0.088 \pm 0.004 \mathrm{~Hz}$, Dox off $(n=229$ neurons, four fields from two mice); $0.074 \pm 0.001 \mathrm{~Hz}$, Dox re-on $\left(n=211\right.$ neurons, four fields from two mice); $0.063 \pm 0.001 \mathrm{~Hz}, F_{(2,602)}=29.19, p=$ $5.83 \times 10^{-5}$ (Dox on vs Dox off), $p=5.15 \times 10^{-4}$ (Dox off vs Dox re-on), $p=9.56 \times 10^{-10}$ (Dox on vs Dox re-on), one-way ANOVA followed by Tukey's test]. Data in panel $\boldsymbol{B}$ are presented as the mean \pm SEM, while data in panels $\boldsymbol{D}, \boldsymbol{E}, \boldsymbol{G}, \boldsymbol{H}$ show means and distributions of each data point; $* p<0.05$, $* * * p<0.001$, n.s.: non-significant.

particularly for neurons located close to each other. To test whether the reduction of C.C. on microglial recovery recovered once the microglial population was restored, we conducted an additional experimental cohort where we tracked neuronal activity and C.C. in the same mice before Dox-off, after $7 \mathrm{~d}$ Dox off, and again after returning the mice to Dox-on chow for $42 \mathrm{~d}$. The density of microglia completely recovered with even just one week of Dox re-administrating $\left[135.0 \pm 14.5 / \mathrm{mm}^{2}(n=6\right.$ fields from three mice); Fig. $3 A, B]$. As shown in Figure $3 G, H$, there was again a significant reduction of C.C. on Dox-off, which partially recovered following Dox return [Fig. 3G; $F_{(2,4926)}=200.21, p=9.56 \times 10^{-10}$ (Dox on: $0.243 \pm$ 0.006 vs Dox off: $0.115 \pm 0.003), p=3.15 \times 10^{-7}$ (Dox off: $0.115 \pm 0.003$ vs Dox re-on: $0.145 \pm 0.005), p=9.56$ $\times 10^{-10}$ (Dox on: $0.243 \pm 0.006$ vs Dox re-on: $0.145 \pm$ 0.005), one-way ANOVA followed by Tukey's test]. In this cohort, there was also a modest (but significant) decrease in neuronal firing frequency after Dox off, which further decreased slightly after $42 \mathrm{~d}$ of Dox return [Fig. 3H; Dox on ( $n=165$ neurons, four fields from two mice); $0.088 \pm$ $0.004 \mathrm{~Hz}$, Dox off ( $n=229$ neurons, four fields from two mice); $0.074 \pm 0.001 \mathrm{~Hz}$, Dox re-on $(n=211$ neurons, four fields from two mice); $0.063 \pm 0.001 \mathrm{~Hz}, F_{(2,602)}=29.19$, $p=5.83 \times 10^{-5}$ (Dox on vs Dox off), $p=5.15 \times 10^{-4}$ (Dox off vs Dox re-on), $p=9.56 \times 10^{-10}$ (Dox on vs Dox re-on), one-way ANOVA followed by Tukey's test]. Hence, partial microglia ablation results in an apparent irreversible reduction in synchronicity, which we propose results from reduced microglia-synapse contact induced modulation of neural activity.

\section{The impact of microglial-synapse contact on neuronal activity is lost on microglial activation}

We next hypothesized that only surveying, physiologic microglia have the ability to effect neural activity by microglial process contact. To test this we used daily sys- temic injection of LPS, a treatment know to activate microglia (Chen et al., 2014). We confirmed that systemic LPS injections for $4 \mathrm{~d}$ resulted in the expected morphologic changes (Fig. 4A,B) whereas control systemic saline injections did not affect microglial properties (salineinjected mice soma diameter: $40.1 \pm 9.2 \mu \mathrm{m}^{2}, n=58$ cells from three mice, LPS-injected mice; $49.2 \pm 11 \mu \mathrm{m}^{2}$, $n=80$ cells from three mice, $p=4.30 \times 10^{-7}$, unpaired $t$ test). In mice with LPS injection for $4 \mathrm{~d}$ (LPS mice), we still observed contacts between spines and microglial processes (Fig. 5A). When we examined contact frequency in response to an acute single LPS injection, we saw a significant decrease, from $1.17 \pm 0.16$ to $0.76 \pm$ $0.13 / h$ after LPS injections $(n=31$ contacts from four mice; $p=0.0132$, paired $t$ test; Fig. $5 B$ ). As was seen in the control mice, a single microglial process in LPSinjected mice contacted different spines, and the same spine was observed to be contacted by different microglial process (Figs. 2, 5A ). However in LPS mice, the microglia-spine contacts had no effect on the frequency of spine $\mathrm{Ca}^{2+}$ transients as compared to the basal rates before or after contact [Fig. 4C-E; $0.013 \pm 0.003 \mathrm{~Hz}$ (during) vs $0.012 \pm 0.003 \mathrm{~Hz}$ (before), $p=0.816, n=6$ fields from three mice (Fig. 4D); $0.013 \pm 0.004 \mathrm{~Hz}$ (during) vs $0.014 \pm 0.005 \mathrm{~Hz}$ (after), $p=0.843, n=6$ fields from three mice (Fig. $4 E$ ), paired $t$ test]. We again imaged $\mathrm{Ca}^{2+}$ transients across multiple neurons in L2/3 of the primary motor cortex (Fig. 4F) and plotted the C.C. against the distance between each of the neurons within the pair, as done above with the Dox on and Dox off mice (Fig. 4G-L). In the 4 and $9 \mathrm{~d}$ saline-injected control mice, there was a negative slope correlation between neuronal separation distance and C.C., as expected, indicating that neurons which were located close to each other were more likely to fire in synchrony [control (4 d injection); $r=-0.135, p=$ $4.45 \times 10^{-47}$ (Fig. $\left.4 /\right)$; control (9 d injection); $r=-0.303$, 
A
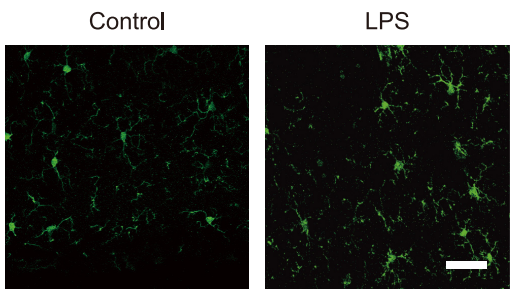

C
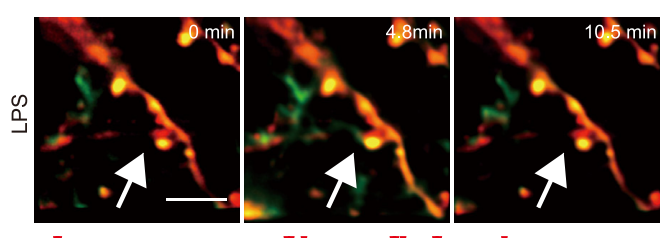

D

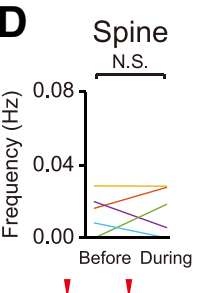

$E$
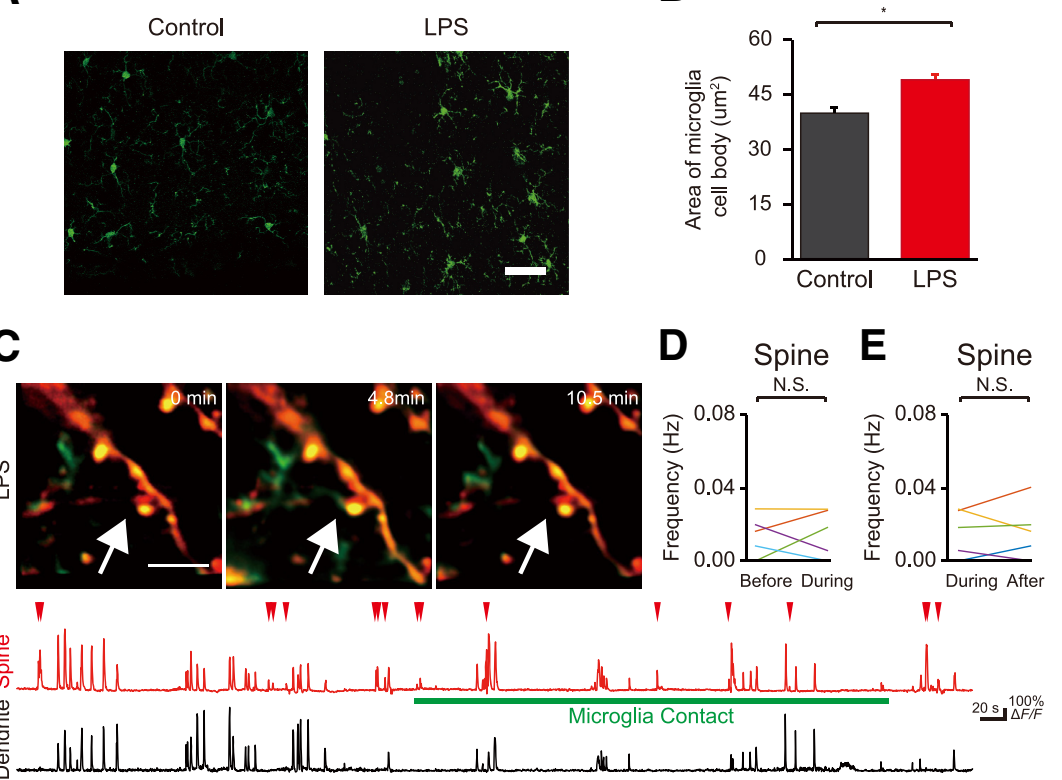

F
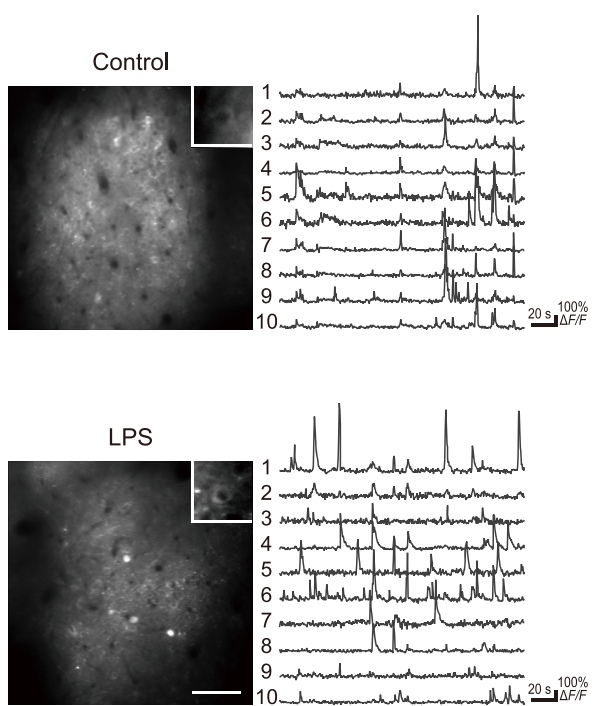

4 days injection

Control
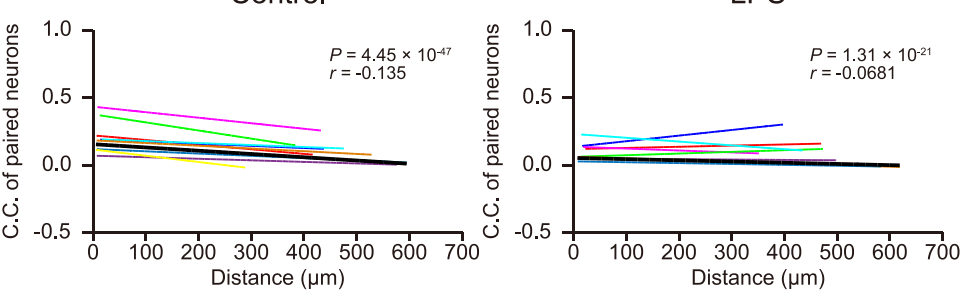

L

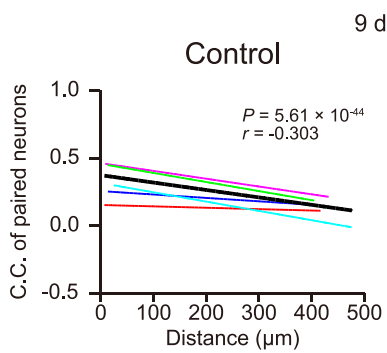

9 days injection

K

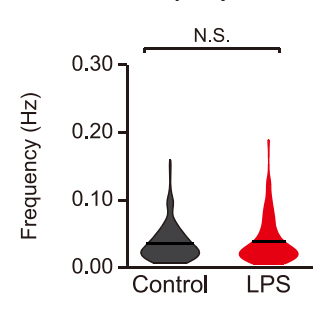

N

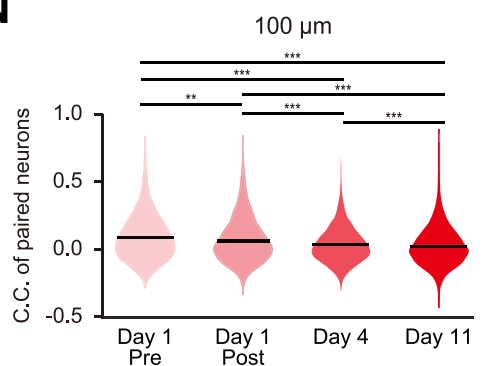

LPS

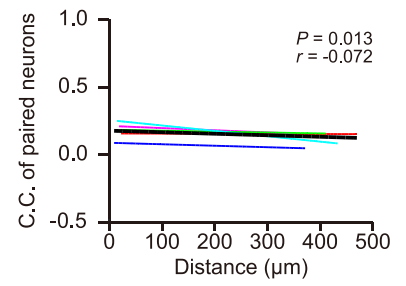

0

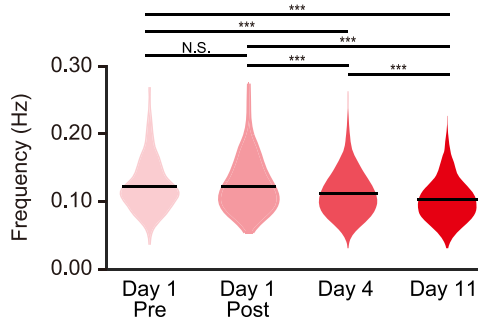

Figure 4. Microglia-synapse interactions in mice following LPS injections. $\boldsymbol{A}, \boldsymbol{B}$, Intraperitoneal injection of LPS activated microglia, as verified by morphologic appearance $(\boldsymbol{A})$, which included a significant increase in soma diameter $(\boldsymbol{B})$. Soma area increased from 40.1 $\pm 9.2 \mu \mathrm{m}^{2}$ in saline-injected mice ( $n=58$ cells from three mice) to $49.2 \pm 11 \mu \mathrm{m}^{2}$ in LPS-injected mice $(n=80$ cells from three mice, $p=4.30 \times 10^{-7}$, unpaired $t$ test). $\boldsymbol{C}$, top, Fluorescent image of a dendrite in L2/3 of the primary motor cortex of an LPS-injected mice showing the simultaneous imaging of spines (tdTomato; red) and microglia (EGFP; green). Scale bar: $5 \mu \mathrm{m}$. As in Figure 1, the lower trace shows a sample recording of $\mathrm{Ca}^{2+}$ transients (GCaMP6f fluorescence $\Delta F / F$ ) in a single spine (upper) and in the parent dendrite (lower) before, during, and after microglial contact. Local synaptic activity was not effected by microglial contact. The white arrow 


\section{continued}

indicate the spine contacted by microglia process. $\boldsymbol{D}, \boldsymbol{E}$, The frequency of $\mathrm{Ca}^{2+}$ transients in spines during the period of microglial contact was not significantly different from that either before $(\boldsymbol{D})$ or after $(\boldsymbol{E})$ contact. Each line shows data from a single experiment [D: $0.013 \pm 0.003 \mathrm{~Hz}$ (during) vs $0.012 \pm 0.003 \mathrm{~Hz}$ (before), $p=0.816, n=6$ fields from three mice; $\boldsymbol{E}: 0.013 \pm 0.004 \mathrm{~Hz}$ (during) vs $0.014 \pm 0.005 \mathrm{~Hz}$ (after), $p=0.843, n=6$ fields from three mice, paired $t$ test]. $\boldsymbol{F}$, Typical fields of view of neurons with GCaMP6f fluorescence in L2/3 of the primary motor cortex of saline-injected mice (upper) and LPS-injected mice (lower), with accompanying representative $\Delta F / F \mathrm{Ca}^{2+}$ traces from ten individual neurons in each mouse. $\mathbf{G}-\boldsymbol{L}$, Distribution of C.C.s $(\boldsymbol{G}, \boldsymbol{J})$ and of neuronal firing frequencies $(\boldsymbol{H}, \boldsymbol{K})$ in mice injected with either saline or LPS for $4 \mathrm{~d}(\boldsymbol{G}, \boldsymbol{H})$ or $9 \mathrm{~d}(\boldsymbol{J}, \boldsymbol{K})$. LPS injections decreased the C.C. for pairs of closely located neurons but had no effect on neuronal firing frequencies. Panels $\boldsymbol{I}, \boldsymbol{L}$ show the relationship between $C$.C. and neuronal separation for mice injected with either saline or LPS for $4 \mathrm{~d}(\boldsymbol{I})$ or $9 \mathrm{~d}(\boldsymbol{L})$. Each line shows the slope of the correlation from individual mice, while the values in the top right corner show the slope of the fits to the complete data set (and the value of the Pearson's correlation test), the fit to this grouped data are shown as the solid dark line. Averaging the individual fits gives a mean slope correlation of: $r=-0.149 \pm 0.019$ for $4 \mathrm{~d}$ saline mice $\left(p=5.43 \times 10^{-5}\right.$, one-sample $t$ test, $n=9$ mice), $r=-0.008 \pm 0.039$ for 4 d LPS mice ( $p=0.850, n=9$ mice), $r=-0.285 \pm 0.065$, for 9 d saline $(p=0.012, n=5$ mice), and $r=-0.107 \pm 0.048$ for 9 d LPS $[p=0.091, n=5$ mice; G: saline-injected mice (4 d injection, $n=9$ mice); $0.12 \pm 0.004$, LPS-injected mice (4 d injection, $n=9$ mice); $0.043 \pm 0.002, p=7.02 \times 10^{-72}$, Wilcoxon rank sum test; $\boldsymbol{H}$ : control (4 d injection, $n=464$ neurons from nine mice); $0.098 \pm 0.002$ $\mathrm{Hz}$, LPS (4 d injection, $n=568$ neurons from nine mice); $0.098 \pm 0.002 \mathrm{~Hz}, p=0.841$, Wilcoxon rank sum test; $\boldsymbol{l}$; control (4 d injection); $r=-0.135, p=4.45 \times 10^{-47}$, LPS (4 d injection); $r=-0.0681, p=1.31 \times 10^{-21}$, Pearson's correlation test; $\boldsymbol{J}$; saline-injected mice (9 d injection, $n=5$ mice); $0.34 \pm 0.008$, LPS-injected mice ( 9 d injection, $n=5$ mice); $0.18 \pm 0.009, p=3.61 \times 10^{-30}$, Wilcoxon rank sum test; $\boldsymbol{K}$; saline-injected mice ( $9 \mathrm{~d}$ injection, $n=141$ neurons from five mice); $0.033 \pm 0.002 \mathrm{~Hz}$, LPS-injected mice ( $9 \mathrm{~d}$ injection, $n=108$ neurons from five mice); $0.037 \pm 0.003 \mathrm{~Hz}, p=0.60$, Wilcoxon rank sum test; $L$; control (9 d injection); $r=-0.303$, $p=5.61 \times 10^{-44}$, LPS (9 d injection); $r=-0.072, p=0.013$, Pearson's correlation test]. Hence LPS injection abolished the correlation between C.C. and neuronal separation. $\mathbf{M - O}$, In an additional cohort of mice, L2/3 neurons were imaged before LPS $<$ and again 1 and $4 \mathrm{~d}$ after daily LPS injections, and then after $7 \mathrm{~d}$ of recovery from the last LPS injection $(\boldsymbol{M})$. The distributions of the C.C. $(\boldsymbol{N})$ and the neuronal firing frequencies $(\mathbf{O})$ over these time periods are shown. LPS injection caused a significant decrease in C.C., which was evident by the first day of injection and did not recover following a week after the last LPS injection. (N). From $4 \mathrm{~d}$ after LPS, the firing frequency in this mouse cohort decreased, and there was a further decrease by one week after the last LPS injection [O; $\boldsymbol{N}: F_{(3,19984)}$ $=189.29, p=0.002$ (day 1 pre; $0.089 \pm 0.004$ vs day 1 post; $0.074 \pm 0.003$ ), $p=3.77 \times 10^{-9}$ (day 1 pre; $0.089 \pm 0.004$ vs day $4 ; 0.035 \pm 0.002), p=3.77 \times 10^{-9}$ (day 1 pre; $0.089 \pm 0.004$ vs day $\left.11 ; 0.023 \pm 0.001\right), p=3.77 \times 10^{-9}$ (day 1 post; $0.074 \pm 0.003$ vs day $4 ; 0.035 \pm 0.002), p=3.77 \times 10^{-9}$ (day 1 post; $0.074 \pm 0.003$ vs day $\left.11 ; 0.023 \pm 0.001\right), p=1.93 \times 10^{-9}$ (day $4 ; 0.035 \pm$ 0.002 vs day $11 ; 0.023 \pm 0.001)$, one-way ANOVA followed by Tukey's test; $\mathbf{O}$ : day 1 pre $(n=320$ neurons, seven fields from four mice); $0.122 \pm 0.002 \mathrm{~Hz}$, day 1 post $(n=325$ neurons, seven fields from four mice); $0.122 \pm 0.002 \mathrm{~Hz}$, day 4 ( $n=449 \mathrm{neurons}$, seven fields from four mice); $0.112 \pm 0.002 \mathrm{~Hz}$, day $11\left(n=737\right.$ neurons, seven fields from four mice); $0.102 \pm 0.001 \mathrm{~Hz}, F_{(3,1827)}=37.13$, $p=1.00$ (day 1 pre vs day 1 post), $p=2.43 \times 10^{-4}$ (day 1 pre vs day 4 ), $p=3.77 \times 10^{-9}$ (day 1 pre vs day 11 ), $p=1.54 \times 10^{-4}$ (day 1 post vs day 4 ), $p=3.77 \times 10^{-9}$ (day 1 post vs day 11), $p=5.09 \times 10^{-5}$ (day 4 post vs day 11), one-way ANOVA followed by Tukey's test]. Data in $\boldsymbol{B}$ shows the mean \pm SEM, the dark lines in panels $\boldsymbol{G}, \boldsymbol{H}, \boldsymbol{J}, \boldsymbol{K}, \boldsymbol{N}, \boldsymbol{O}$ represent the means; $* p<0.05, * * p$ $<0.01, * * * p<0.001$, n.s.: non-significant.

$p=5.61 \times 10^{-44}$ (Fig. 4L), Pearson's correlation test]. We compared these values for 4 and $9 \mathrm{~d}$ saline-injected mice with those of 4 and $9 \mathrm{~d}$ LPS-injected mice. LPS injection, for either 4 or $9 \mathrm{~d}$, significantly reduced the C.C. and decreased the extent of the relationship between neuronal separation and C.C. [Fig. 4G,I,J,L; saline-injected mice (4 $\mathrm{d}$ injection, $n=9$ mice); $0.12 \pm 0.004$, LPS-injected mice (4 d injection, $n=9$ mice); $0.043 \pm 0.002, p=7.02 \times$ $10^{-72}$ (Fig. 4G); LPS (4 d injection); $r=-0.0681, p=1.31$ $\times 10^{-21}$ (Fig. 4l); saline-injected mice ( 9 d injection, $n=5$ mice); $0.34 \pm 0.008$, LPS-injected mice ( $9 \mathrm{~d}$ injection, $n=$ 5 mice); $0.18 \pm 0.009, p=3.61 \times 10^{-30}$, Wilcoxon rank sum test (Fig. 4J); LPS (9 d injection); $r=-0.072, p=$ 0.013, Pearson's correlation test (Fig. 4L)]. In contrast, LPS treatment did not significantly impact on neuronal firing frequency [Fig. $4 H, K$; control $(4 \mathrm{~d}, n=464$ from nine mice); $0.098 \pm 0.002 \mathrm{~Hz}$, LPS (4 d, $n=568$ from nine mice); $0.098 \pm 0.002 \mathrm{~Hz}, p=0.841$ (Fig. $4 H$ ); control (9 d, $n=141$ from five mice); $0.033 \pm 0.002 \mathrm{~Hz}$, LPS (9 d, $n=$ 108 from five mice); $0.037 \pm 0.003 \mathrm{~Hz}, p=0.60$ (Fig. $4 K$ ), Wilcoxon rank sum test]. Hence the synchronized firing of neurons, and particularly for closely located neurons, is abolished by LPS-induced microglial activation.

We further investigated the time course and recovery profile for the effects of LPS injections, using four mice which received LPS injections over $4 \mathrm{~d}$ with repeated imaging, with the final imaging session $7 \mathrm{~d}$ after stopping LPS injections (Fig. 4M-O). Even after a single LPS injection, there was a significant decrease in the C.C., and this further decreased after $4 \mathrm{~d}$ of LPS injections. Following one week of recovery, the C.C. was still significantly decreased [Fig. $4 N ; F_{(3,19984)}=189.29, p=0.002$ (day 1 pre; $0.089 \pm 0.004$ vs day 1 post; $0.074 \pm 0.003), p=$ $3.77 \times 10^{-9}$ (day 1 pre; $0.089 \pm 0.004$ vs day $4 ; 0.035 \pm$ $0.002), p=3.77 \times 10^{-9}$ (day 1 pre; $0.089 \pm 0.004$ vs day $11 ; 0.023 \pm 0.001), p=3.77 \times 10^{-9}$ (day 1 post; $0.074 \pm$ 0.003 vs day $4 ; 0.035 \pm 0.002$ ), $p=3.77 \times 10^{-9}$ (day 1 post; $0.074 \pm 0.003$ vs day $11 ; 0.023 \pm 0.001), p=1.93$ $\times 10^{-9}$ (day $4 ; 0.035 \pm 0.002$ vs day $11 ; 0.023 \pm 0.001$ ), one-way ANOVA followed by Tukey's test]. This lack of recovery of C.C. is consistent with the persistent (>28 d) activation of microglia induced by a single LPS injection (Kondo et al., 2011). The neuronal firing frequency did not change after a single LPS injection. However, in contrast to what we had observed with the 4 and $9 \mathrm{~d}$ treatment cohorts above, the firing frequency continued to decrease slightly over each of the subsequent imaging days [Fig. 40; day 1 pre ( $n=320$ neurons, seven fields from four mice); $0.122 \pm 0.002 \mathrm{~Hz}$, day 1 post $(n=325$ neurons, seven fields from four mice); $0.122 \pm 0.002 \mathrm{~Hz}$, day 4 ( $n=$ 
A
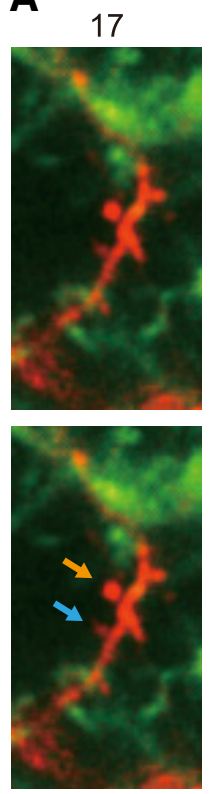

52
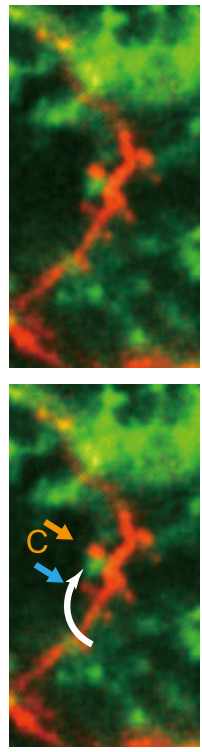

83
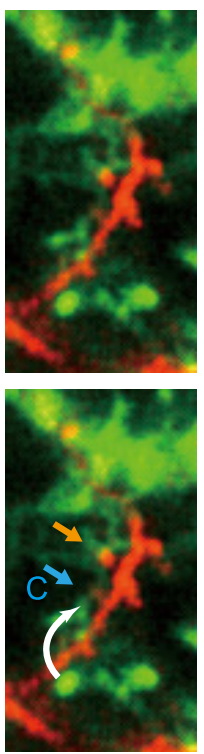

125
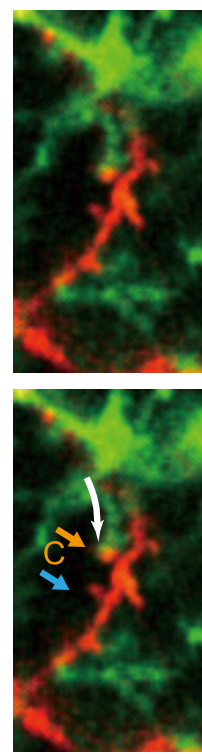

B
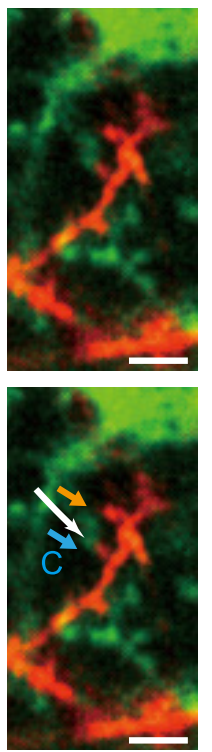

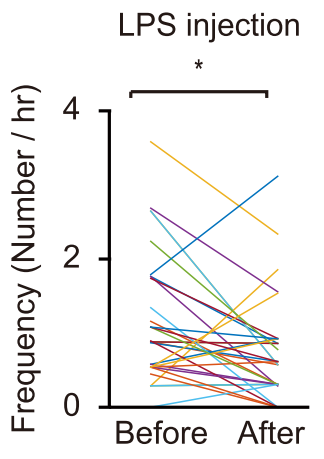

Figure 5. Specific activated microglial processes contact different spines, while a single spine may be contacted by different activated microglia. $\boldsymbol{A}$, As in Figure 2 for physiologic microglia, the processes of the same specific activated microglia contact two different spines (orange arrow spine at $52 \mathrm{~min}$, blue arrow spine at $83 \mathrm{~min}$ ). The same two spines are later contacted by processes originating from a different microglia. $\boldsymbol{B}$. The mean frequency of contacts between microglial processes and spines in mice with an acute injection of LPS. LPS decreases the contact frequency from $1.17 \pm 0.16 / \mathrm{h}$ under control to $0.76 \pm 0.13 / \mathrm{h}$ after LPS injection $(n=31$ spines from four mice, $p=0.0132$, paired $t$ test). $* p<0.05$.

449 neurons, seven fields from four mice); $0.112 \pm 0.002$ $\mathrm{Hz}$, day 11 ( $n=737$ neurons, seven fields from four mice); $0.102 \pm 0.001 \mathrm{~Hz}, F_{(3,1827)}=37.13, p=1.00$ (day 1 pre vs day 1 post), $p=2.43 \times 10^{-4}$ (day 1 pre vs day 4 ), $p=3.77$ $\times 10^{-9}$ (day 1 pre vs day 11 ), $p=1.54 \times 10^{-4}$ (day 1 post vs day 4$), p=3.77 \times 10^{-9}$ (day 1 post vs day 11$), p=$ $5.09 \times 10^{-5}$ (day 4 post vs day 11 ), one-way ANOVA followed by Tukey's test].

\section{Discussion}

Microglial undertake continuous surveillance of neurons in healthy and diseased brain (Nimmerjahn et al., 2005; Wake et al., 2009). These microglia-neuron contacts can help sculpt the structural elements of neural circuits through formation (Parkhurst et al., 2013; Miyamoto et al., 2016) and elimination (Schafer et al., 2012) of synapses. We show here that the brief contacts between microglial processes and synapses in awake, healthy adult mice result in an acute and highly localized functional change in neural activity. Contact was followed by more $\mathrm{Ca}^{2+}$ transients in dendritic spines, reflecting enhanced synaptic activity. Such effects were only seen for physiologic microglia: microglia activated by LPS injections still contacted spines but did not impart on them increases in activity. Furthermore, the extent to which activity in spatially close L2/3 neurons was synchronized was also reduced following microglia activation by LPS, or following partial microglial ablation. We propose therefore that the functional consequences of the local increase in activity imparted by contact with processes of physiologic microglia is to enhance microscopic synchronization.
The LPS induced reduction in synchrony was evident after a single LPS injection, and further decreased when monitored after $4 \mathrm{~d}$ LPS injection. A single LPS injection $(0.5 \mathrm{mg} / \mathrm{kg}$, i.p.) has been shown to cause a sustained activation of microglia (for at least $28 \mathrm{~d}$; Kondo et al., 2011) and the same $4 \mathrm{~d}$ LPS injection protocol $(1 \mathrm{mg} / \mathrm{kg}$, i.p.) causes a marked activation of microglia (Chen et al., 2014). The inhibition of synchronous firing of neurons was still reduced (and even more strongly inhibited) at $7 \mathrm{~d}$ following the last LPS injection, suggesting that sustained microglia activation may continue to inhibit neuronal synchronicity. Alternatively, a lack of microglia-induced synchronization may have a prolonged effect, as even when microglial numbers had returned to control levels following a transient ablation (in the Dox-off mice) the extent of synchrony only partially recovered. It is not known, however, whether microglia repopulating the brain following an ablation return immediately to their original physiologic phenotype. Chen et al., reported that sustained LPSinduced microglia activation was associated with "synaptic stripping" of inhibitory terminals on Layer III and V neurons in the motor cortex, that was associated with enhanced gamma band power in local field potential recordings (Chen et al., 2014). Our direct $\mathrm{Ca}^{2+}$ imaging revealed no increases in activity levels, if anything these tended to decrease after LPS injections or microglial ablation. Clearly, brain inflammation can impart effects on neuronal activity and cognitive processing via two broad mechanistic pathways: effects arising from a loss of physiologic microglia induced neuronal synchrony, and effects 
arising from changes in synapses and circuits due to consequences of activated microglia.

Coordinated neuronal activity is a fundamental component of brain activity which may arise from underlying oscillations or from temporally related sensory or motor responses. At the macroscopic level, neurons within cortical columns respond with some synchrony during specific sensory or motor modalities. However, at a more microscopic level, in vivo imaging of cortical activity has also demonstrated localized patterns of synchronous neural activity. Subsets of motor cortical L2/3 neurons within close $(\approx<100 \mu \mathrm{m})$ proximity, for example, respond synchronously during running, grooming or lever pulling (Dombeck et al., 2009; Hira et al., 2013). Shared excitatory cortical afferent inputs (Yoshimura et al., 2005) and/or synaptic interconnections between closely co-localized neuron pairs (Ko et al., 2011) may contribute to such microscopic L2/3 neuron synchronicity. In addition to these "wiring" mechanisms, we suggest that physiologic microglia can also help entrain pyramidal neurons into a microscopic or local synchronized circuit. The restriction of synchronization to close proximity aligns with the surveillance domain of the processes of a single microglia $(<50 \mu \mathrm{m}$; Nimmerjahn et al., 2005). We propose that subsets of synapses within the domains of each microglia are synchronized. How these processes know which neurons are to be recruited into a neural circuit associated with a particular modality is unclear, but neuronal activity can attract microglial contact (Li et al., 2012). It may be that neurons participating most strongly in a particular response preferentially attract microglial process, which then further enhance the activity of these neurons and thereby help synchronize these neurons into a circuit or engram.

The functional implications of microglial-nerve contacts in synchronizing local circuits we report here were derived from male mice. A number of gender differences in microglial dynamics and responses have been reported. As reviewed by Rahimian et al. (2018), the morphology and phagocytic phenotypes of male and female microglia differ over development, and gender differences in the inflammatory response to ischemia have also been observed in adult brain. Whether gender differences in the physiologic microglia-neuron interactions we report here for adults also exist is unclear, but will be of interest to consider in light of the gender differences in inflammation-associated risk factors for schizophrenia and in the incidence of autism spectrum disorders (Lenz and McCarthy, 2015).

Our result has potential implications for understanding how immune system activation may impact on cognitive function. Maternal infection and chronic inflammatory disorders in adults (e.g., periodontitis) are associated with an increase incidence of brain disorders characterized by cognitive impairments (Noble et al., 2009; Knuesel et al., 2014), while some genetic brain disorders have microglial genes as their proposed basis (see Wake et al., 2013). We have previously speculated that microglial activation and/or dysfunction may be causative for cognitive disorders (Wake et al., 2013), and our current results suggests an additional putative mechanistic link for such an association. Further understanding of the signaling pathways and behavioral consequences of this enhancement of synaptic activity by physiologic microglial surveillance may provide insights to address cognitive disorders involving altered immune and microglial status.

\section{References}

Chen Z, Jalabi W, Hu W, Park HJ, Gale JT, Kidd GJ, Bernatowicz R, Gossman ZC, Chen JT, Dutta R, Trapp BD (2014) Microglial displacement of inhibitory synapses provides neuroprotection in the adult brain. Nat Commun 5:4486. CrossRef Medline

Cunningham C (2013) Microglia and neurodegeneration: the role of systemic inflammation. Glia 61:71-90. CrossRef Medline

Dissing-Olesen L, LeDue JM, Rungta RL, Hefendehl JK, Choi HB, MacVicar BA (2014) Activation of neuronal NMDA receptors triggers transient ATP-mediated microglial process outgrowth. J Neurosci 34:10511-10527. CrossRef

Dombeck DA, Khabbaz AN, Collman F, Adelman TL, Tank DW (2007) Imaging large-scale neural activity with cellular resolution in awake, mobile mice. Neuron 56:43-57. CrossRef Medline

Dombeck DA, Graziano MS, Tank DW (2009) Functional clustering of neurons in motor cortex determined by cellular resolution imaging in awake behaving mice. J Neurosci 29:13751-13760. CrossRef

Estes ML, McAllister AK (2015) Immune mediators in the brain and peripheral tissues in autism spectrum disorder. Nat Rev Neurosci 16:469-486. CrossRef Medline

Eyo UB, Peng J, Swiatkowski P, Mukherjee A, Bispo A, Wu LJ (2014) Neuronal hyperactivity recruits microglial processes via neuronal NMDA receptors and microglial P2Y12 receptors after status epilepticus. J Neurosci 34:10528-10540. CrossRef

Hira R, Ohkubo F, Ozawa K, Isomura Y, Kitamura K, Kano M, Kasai H, Matsuzaki M (2013) Spatiotemporal dynamics of functional clusters of neurons in the mouse motor cortex during a voluntary movement. J Neurosci 33:1377-1390. CrossRef

Hirasawa T, Ohsawa K, Imai Y, Ondo Y, Akazawa C, Uchino S, Kohsaka S (2005) Visualization of microglia in living tissues using Iba1-EGFP transgenic mice. J Neurosci Res 81:357-362. CrossRef Medline

Kettenmann H, Hanisch UK, Noda M, Verkhratsky A (2011) Physiology of microglia. Physiol Rev 91:461-553. CrossRef Medline

Kipnis J (2016) Multifaceted interactions between adaptive immunity and the central nervous system. Science 353:766-771. CrossRef

Knuesel I, Chicha L, Britschgi M, Schobel SA, Bodmer M, Hellings JA, Toovey S, Prinssen EP (2014) Maternal immune activation and abnormal brain development across CNS disorders. Nat Rev Neurol 10:643-660. CrossRef Medline

Ko H, Hofer SB, Pichler B, Buchanan KA, Sjöström PJ, Mrsic-Flogel TD (2011) Functional specificity of local synaptic connections in neocortical networks. Nature 473:87-91. CrossRef Medline

Kondo S, Kohsaka S, Okabe S (2011) Long-term changes of spine dynamics and microglia after transient peripheral immune response triggered by LPS in vivo. Mol Brain 4:27. CrossRef Medline

Lenz KM, McCarthy MM (2015) A starring role for microglia in brain sex differences. Neuroscientist 21:306-321. CrossRef

Li Y, Du XF, Liu CS, Wen ZL, Du JL (2012) Reciprocal regulation between resting microglial dynamics and neuronal activity in vivo. Dev Cell 23:1189-1202. CrossRef Medline

Masamizu Y, Tanaka YR, Tanaka YH, Hira R, Ohkubo F, Kitamura K, Isomura Y, Okada T, Matsuzaki M (2014) Two distinct layerspecific dynamics of cortical ensembles during learning of a motor task. Nat Neurosci 17:987-994. CrossRef Medline

Miyamoto A, Wake $\mathrm{H}$, Ishikawa AW, Eto K, Shibata K, Murakoshi $\mathrm{H}$, Koizumi S, Moorhouse AJ, Yoshimura Y, Nabekura J (2016) Microglia contact induces synapse formation in developing somatosensory cortex. Nat Commun 7:12540. CrossRef Medline 
Nimmerjahn A, Kirchhoff F, Helmchen F (2005) Resting microglial cells are highly dynamic surveillants of brain parenchyma in vivo. Science 308:1314-1318. CrossRef

Noble JM, Borrell LN, Papapanou PN, Elkind MS, Scarmeas N, Wright CB (2009) Periodontitis is associated with cognitive impairment among older adults: analysis of NHANES-III. J Neurol Neurosurg Psychiatry 80:1206-1211. CrossRef Medline

Parkhurst CN, Yang G, Ninan I, Savas JN, Yates JR 3rd, Lafaille JJ, Hempstead BL, Littman DR, Gan WB (2013) Microglia promote learning-dependent synapse formation through brain-derived neurotrophic factor. Cell 155:1596-1609. CrossRef

Rahimian R, Cordeau P Jr, Kriz J (2018) Brain response to injuries: when microglia go sexist. Neuroscience pii: S0306-4522: 3017330178. CrossRef Medline

Salter MW, Beggs S (2014) Sublime microglia: expanding roles for the guardians of the CNS. Cell 158:15-24. CrossRef Medline

Schafer DP, Lehrman EK, Kautzman AG, Koyama R, Mardinly AR, Yamasaki R, Ransohoff RM, Greenberg ME, Barres BA, Stevens B (2012) Microglia sculpt postnatal neural circuits in an activity and complement-dependent manner. Neuron 74:691-705. CrossRef

Stanger BZ, Tanaka AJ, Melton DA (2007) Organ size is limited by the number of embryonic progenitor cells in the pancreas but not the liver. Nature 445:886-891. CrossRef Medline
Tanaka KF, Matsui K, Sasaki T, Sano H, Sugio S, Fan K, Hen R, Nakai J, Yanagawa Y, Hasuwa H, Okabe M, Deisseroth K, Ikenaka K, Yamanaka A (2012) Expanding the repertoire of optogenetically targeted cells with an enhanced gene expression system. Cell Rep 2:397-406. CrossRef Medline

Tay TL, Savage J, Hui CW, Bisht K, Tremblay ME (2017) Microglia across the lifespan: from origin to function in brain development, plasticity and cognition. J Physiol 595:1929-1945.

Thevenaz P, Ruttimann UE, Unser M (1998) A pyramid approach to subpixel registration based on intensity. IEEE Trans Image Process 7:27-41. CrossRef

Tremblay MĖ, Lowery RL, Majewska AK (2010) Microglial interactions with synapses are modulated by visual experience. PLoS Biol 8:e1000527. CrossRef Medline

Wake H, Moorhouse AJ, Jinno S, Kohsaka S, Nabekura J (2009) Resting microglia directly monitor the functional state of synapses in vivo and determine the fate of ischemic terminals. J Neurosci 29:3974-3980. CrossRef

Wake H, Moorhouse AJ, Miyamoto A, Nabekura J (2013) Microglia: actively surveying and shaping neuronal circuit structure and function. Trends Neurosci 36:209-217. CrossRef Medline

Yoshimura Y, Dantzker JL, Callaway EM (2005) Excitatory cortical neurons form fine-scale functional networks. Nature 433:868-873. CrossRef Medline 